\title{
Reduced thermal hyperalgesia and enhanced peripheral nerve injury after hind paw inflammation in mice lacking the serotonin-transporter
}

\author{
Florian Palm ${ }^{\mathrm{a}}$, Rainald Mössner ${ }^{\mathrm{b}}$, Yong Chen ${ }^{\mathrm{a}}$, Lan $\mathrm{He}^{\mathrm{a}}$, Manfred Gerlach ${ }^{\mathrm{b}}$, Stefan \\ Bischofs $^{\mathrm{a}}$, Peter Riederer ${ }^{\mathrm{b}}$, Klaus-Peter Lesch $^{\mathrm{b}}{ }^{\text {, Claudia Sommer }}{ }^{\mathrm{a}, *}$ \\ a Department of Neurology, University of Würzburg, Neurologische Klinik der Universität, D-97080 Würzburg, Germany \\ ${ }^{\mathrm{b}}$ Department of Psychiatry and Psychotherapy, University of Würzburg, D-97080 Würzburg, Germany
}

Received 27 August 2007; received in revised form 2 November 2007; accepted 29 November 2007

Available online 9 January 2008

\begin{abstract}
Mice lacking the serotonin-transporter (5-HTT-/- mice) develop reduced thermal hyperalgesia after nerve injury, concomitant with reduced serotonin (5-HT) levels in nervous tissue. Here we investigated pain behaviour in 5-HTT-/- mice compared to their wild type littermates after hind paw inflammation induced by complete Freund's adjuvant (CFA). We used standard tests for pain behaviour, high performance liquid chromatography for measurement of 5-HT, and immunohistochemistry of hind paw skin tissue and L5 dorsal root ganglia (DRG) to measure local inflammation and nerve injury. After intraplantar CFA injection, hyperalgesia to heat was attenuated in 5-HTT-/- mice compared to wild type mice. Their 5-HT levels in nervous and adrenal tissue were reduced. An intraplantar injection of 5-HT four days after CFA transiently brought withdrawal latencies of 5-HTT-/- mice down to the level of wild type mice, thus rescuing the phenotype and supporting the role of 5-HT in the development of CFA-induced thermal hyperalgesia. The density of intraepidermal nerve fibres in plantar skin after CFA injection was reduced to a higher degree in 5-HTT-/mice than in wild type mice, suggesting greater peripheral nerve injury in the knock-out mice during hind paw inflammation. Accordingly, a higher number of injured DRG neurons was identified by activating transcription factor 3 (ATF3) staining in 5HTT-/- mice after CFA. We conclude that the phenotype of 5-HTT-/- mice leads to reduced inflammatory pain due to reduced tissue 5-HT levels and to greater peripheral nerve injury after inflammation. Human variants of the 5-HTT genotypes might be part of the factors determining the extent of nerve injury and hyperalgesia in inflammation.

(C) 2007 European Federation of Chapters of the International Association for the Study of Pain. Published by Elsevier Ltd. All rights reserved.
\end{abstract}

Keywords: Serotonin; Serotonin-transporter; ATF3; Intraepidermal nerve fibres; Inflammatory pain

\section{Introduction}

Serotonin (5-HT) has important functions in pain processing and modulation (Eide and Hole, 1993). In the periphery, 5-HT is released from platelets and mast

\footnotetext{
${ }^{*}$ Corresponding author. Tel.: +49 931201 23763; fax: +49931201 23697.

E-mail address: sommer@mail.uni-wuerzburg.de (C. Sommer).

URL: http://www.uni-wuerzburg.de/neurologie/ (C. Sommer).
}

cells after tissue injury (Dray, 1995) and exerts direct actions on C-fibres (Kessler et al., 1992; Davis et al., 1993; Moalem et al., 2005). The sodium-dependent, high affinity 5-HT transporter (5-HTT) is responsible for the removal of 5-HT from the extracellular space after its release (Lesch et al., 1997; Bengel et al., 1998). Mice with a deficiency of the 5-HT-transporter (5-HTT-/- mice) have increased extracellular 5-HT levels (Fabre et al., 2000; Montanez et al., 2003; Mathews et al., 2004) but the overall tissue content of 5-HT is reduced (Bengel 
et al., 1998; Vogel et al., 2003; Kim et al., 2005). These changes make 5-HTT-/- mice an interesting model to study pain behaviour. We recently investigated 5-HTT-/- mice in a model of an experimental mononeuropathy, the chronic constriction injury of the sciatic nerve (CCI) (Bennett and Xie, 1988; Vogel et al., 2003). We found that 5-HTT-/- mice, in contrast to wild type littermates, did not develop thermal hyperalgesia after CCI. CCI was associated with an increase in 5-HT levels in the sciatic nerve in wild type mice, which was absent in 5-HTT-/- mice. We concluded that 5-HT in the peripheral nerve is necessary for the development of thermal hyperalgesia, thus confirming the role of 5-HT as a factor in the sensitization of nerve fibres. In the present study, we used an inflammatory model, intraplantar (i.pl.) injection of complete Freund's adjuvant (CFA) to further test the role of 5-HT in peripheral sensitization. Peripheral sensitization is the product of the action of inflammatory mediators on nociceptors (McMahon et al., 2005). Thus, the two components have to be present, the sensitizing mediators and functioning C-fibres. Since CFA has been shown to hinder nerve regeneration (Kato et al., 2003), we also investigated whether the i.pl. CFA injections altered the innervation of the hind paw skin and the integrity of the primary sensory neurons. We hypothesised that the functionality of the 5-HTT might be one of the factors determining the extent of hyperalgesia and nerve injury after peripheral inflammation.

\section{Materials and methods}

\subsection{Animals and injections}

Experiments were conducted in adult female mice of $24-37$ g body weight. The mice were generated as previously described on a C57BL/6J background (Bengel et al., 1998) and included homozygous knock-out (5-HTT-/-) and littermate wild type control (5-HTT $+/+)$ mice. Mice were housed individually or in groups of up to five in a temperature and humidity controlled environment, on a 14/10 h light/dark cycle and with standard rodent food pellets and water ad libitum. Experiments were done during the light cycle. All experiments were approved by the Bavarian state authorities and performed in accordance with the European Communities Council Directive of November 24, 1986 (86/609/EEC) for the care and use of laboratory animals.

In short ether anaesthesia, mice received $10 \mu \mathrm{l}$ of CFA, containing $20 \mu \mathrm{g}$ of Mycobacterium tuberculosis (Difco Laboratories, Detroit, Mi, USA) or $10 \mu \mathrm{l}$ of physiological saline into the plantar surface of the right hind paw. Paw swelling was measured with a slide gauge and was used as a sign of effectively induced inflamma- tion (Ferreira et al., 2001; Zhang et al., 2004). Thermal and mechanical sensitivity were tested as described below. An additional group of 5-HTT-/- mice received i.pl. injections into the same area previously injected with CFA with $2 \mu \mathrm{g}$ of 5-HT (Sigma Chemicals, Deisenhofen, Germany) in $5 \mu \mathrm{l}$ of normal saline or normal saline only on day 4 after CFA. In these mice, thermal withdrawal thresholds were additionally tested at 12 and $60 \mathrm{~min}$ and at $24 \mathrm{~h}$ after the 5-HT injection.

\subsection{Behavioural testing}

The investigator was unaware of the genotype. Before the injection of CFA, the mice were tested on two days to determine baseline thresholds. After the injection, the mice were tested at $4 \mathrm{~h}$ and then daily until day seven. Before each test, the animals were allowed to acclimatize for at least $5 \mathrm{~min}$ in the testing device. Both hind paws were tested alternately with an interval of at least 2 min. Mechanical sensitivity was tested with von Frey hairs with bending forces from $4 \mathrm{mg}$ to $1.48 \mathrm{~g}$. The $50 \%$ mechanical withdrawal threshold (the force of the von Frey hair in $\mathrm{g}$ to which an animal reacts in 50\% of the presentations) was determined on the basis of the up-and-down method of Dixon (1965) according to Chaplan et al. (1994), modified for mice (Sommer and Schäfers, 1998). The withdrawal latency to heat stimulation was tested by using a device of Hargreaves et al. (1988) purchased from Ugo Basile (Comerio, Italy). The time until the animals reacted with withdrawal to the stimulation by a radiant heat source was determined automatically. Each testing period consisted of a minimum of three presentations for each hind paw. If the animal did not react within $12 \mathrm{~s}$, the stimulation was stopped manually to prevent tissue damage and the value of $12 \mathrm{~s}$ was assumed.

\subsection{Tissue harvesting}

The tissue was collected from separate groups of mice on day 2 and 7 after injection of CFA under deep barbiturate anaesthesia. At first a piece of each sciatic nerve on both sides with a length of $1 \mathrm{~cm}$ located in the middle of the thigh was harvested. After that the animal was sacrificed by decapitation and the dorsal root ganglia (DRG) L5 were dissected, a piece of the spinal cord (cut just proximally to the L4 and distally to the L5 spinal root), the adrenal glands and three footpads were prepared. The DRGs were embedded in Tissue Tek ${ }^{\circledR}$ (Sakura Finetek, Zoeterwoude, Netherlands), frozen in 2-methylbutan cooled in liquid nitrogen, and stored at $-70{ }^{\circ} \mathrm{C}$. The footpads were fixed in $4 \%$ phosphate-buffered paraformaldehyde ( $\mathrm{pH}$ 7.4) for $3 \mathrm{~h}$, cryoprotected in $10 \%$ sucrose overnight and then frozen as described above. Tissue for 5-HT analysis was weighed and immediately frozen at $-70^{\circ} \mathrm{C}$. 


\subsection{Immunohistochemistry}

Cryosections (10- $\mu \mathrm{m}$ for DRG and spinal cord, $20-\mu \mathrm{m}$ for footpad immunohistochemistry and $40-\mu \mathrm{m}$ for fluorescence) were mounted on Super Frost ${ }^{\circledR}$-Slides from Langenbrinck (Emmerdingen, Germany) and stored at $-20^{\circ} \mathrm{C}$. Immunohistochemistry was performed as previously described (Schäfers et al., 2003) using primary antibodies to activating transcription factor 3 (ATF3,1:200, rabbit anti-human/rat/mouse, Santa Cruz Biotechnology, Santa Cruz, CA, USA), alpha-calcitonin-gene related peptide (CGRP, 1:500, rabbit anti rat/mouse, Peninsula Laboratories, San Carlos, CA, USA), mouse macrophages (MAC-1, 1:100, rat anti-mouse, Camon, Wiesbaden, Germany), and protein gene product (PGP) 9.5 (1:500, rabbit anti-human/rat/mouse Ultra Clone, Wellow, Great Britain). Stains were developed with an ABC method (Vector Laboratories, Burlingame, CA, USA) and $0.02 \%$ diaminobenzidine as chromogen, except for PGP 9.5 and CGRP, which were developed using immunofluorescence with $\mathrm{Cy} 3$ conjugated goat Anti-Rabbit IgG (1:50, Jackson Immuno Research, West Grove, PA, USA).

\subsection{Morphometry}

The fluorescence stains of the footpads were analyzed as described before (Lindenlaub et al., 2000) by using an Axiophot 2 microscope from Zeiss (Oberkochen, Germany), a video camera (DXC 003P) from Sony (Tokyo, Japan), and the software Image Pro Plus 4.0 for Windows 98 from Microsoft (Media Cybernetics, Silver Spring, USA). For determination of intraepidermal nerve fibres (IENF) in PGP 9.5- and CGRP-stains, all fibres in the epidermis in three sections per mouse were counted and then expressed per $\mathrm{mm}$ of analyzed epidermal length. For determination of immunoreactive DRG cells ATF3 stains, the number of immunoreactive nuclei in $\%$ of all neuronal nuclei was determined.

\subsection{Determination of 5-HT}

Samples were taken from the mid sciatic nerve with a length of $1 \mathrm{~cm}$, from the $\mathrm{L} 4 / 5$ spinal cord (cut just proximally to the L4- and distally to the L5-spinal root) and the adrenal glands. These tissues were chosen to have representative samples of the peripheral and central nervous system, the adrenal glands (i.e. non-neuronal serotonergic tissue) served as an internal control. Samples were weighed and frozen at $-70{ }^{\circ} \mathrm{C}$. After thawing, samples were sonicated under argon in ice-cold $150 \mathrm{mM}$ $\mathrm{H}_{3} \mathrm{PO}_{4}$ and $500 \mu \mathrm{M}$ DTPA and centrifuged at $35.000 \mathrm{~g}$ for $20 \mathrm{~min}$ at $+4{ }^{\circ} \mathrm{C}$. The supernatant was filtered through Millipore Ultrafree-MC filter cups at $9.000 \mathrm{~g}$, $4{ }^{\circ} \mathrm{C}$, for $1-2 \mathrm{~h}$, and $50 \mu \mathrm{l}$ of the filtrate was analyzed for 5-HT by reverse-phase HPLC with electrochemical detection. The HPLC system consisted of a AGILENT 1100 series (Bio-Rad, Munich, Germany), a Nucleosil $120-5 \mathrm{C}_{18}$ reverse-phase $(250 \times 4.6 \mathrm{~mm})$ analytical column (Machery-Nagel, Düren, Germany), an electrochemical detector (model 1640; Bio-Rad, Munich, Germany), and a mobile phase (flow rate: $1.5 \mathrm{ml} / \mathrm{min}$ ) containing $0.1 \mathrm{M}$ sodium phosphate buffer, $\mathrm{pH}$ 3.9, $84 \%(\mathrm{v} / \mathrm{v})$ and $16 \%(\mathrm{v} / \mathrm{v})$ methanol containing $0.1 \mathrm{mM}$ EDTA, $0.65 \mathrm{mM}$ 1-octanesulfonic acid and $0.5 \mathrm{mM}$ triethylamine. The detector potential was set at $+0.75 \mathrm{~V}$ relative to the $\mathrm{Ag}-\mathrm{AgCl}$ reference electrode. The signal from the detector was recorded and data analyses were performed using an AGILENT Chem Statian for LC9D (Bio-Rad, Munich, Germany). Concentrations were calculated from the peak height with the aid of an external standard. The investigator was blind to the animals' genotype.

\subsection{Statistical analysis}

For statistical analysis, SPSS (Version 10.0) was used. Results are presented as means $\pm \mathrm{sd}$. To compare behavioural data between groups and test days, a two-way repeated-measures ANOVA was used for parametric analysis, followed by a Student's $t$-test. For nonparametric analysis of the von Frey thresholds, a MannWhitney-U-Test was used for comparison of data between groups. Two-way ANOVA and Student's t test for post hoc analysis were used to compare 5-HT data between the groups. A value of $p<0.05$ was considered to be statistically significant.

\section{Results}

\subsection{5-HTT-/- mice have reduced thermal hyperalgesia after CFA injection}

Baseline values for withdrawal latencies to thermal stimuli and withdrawal threshold to mechanical stimuli, as well as the paw thickness, did not differ between wild type and 5-HTT-/- mice ( $n=8$ each). Both genotypes developed significant paw swelling after i.pl. CFA, which lasted until day 6, without differences between genotypes (Fig. 1A).

Four hours after i.pl. CFA injection, both genotypes showed a significant ipsilateral hypersensitivity to heat. Twenty-four hours after CFA injection, the withdrawal latencies to heat on the ipsilateral side decreased to a minimum in wild type mice, whereas the values in 5HTT-/- mice had already increased again and reached baseline values on day 2 . The withdrawal latencies in saline injected mice and on the contralateral sides (data not shown) of both genotypes did not change compared to baseline over the complete duration of the experiment (Fig. 1B). Ipsilateral withdrawal thresholds to von Frey 

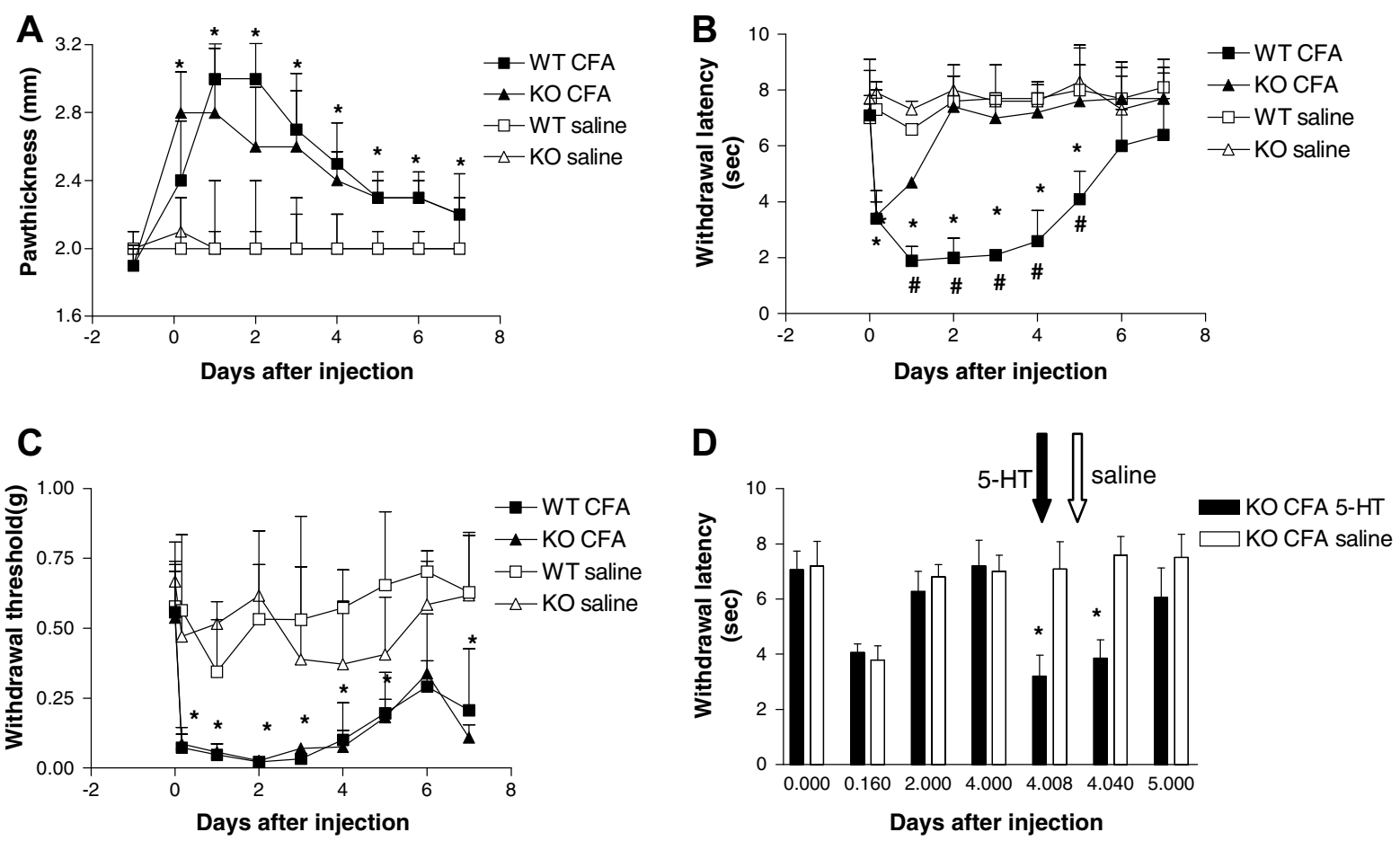

Fig. 1. (A) Mean ipsilateral paw thickness after injection of complete Freund's adjuvant (CFA) or normal saline (saline) in wild type (WT) and 5HTT-/- mice ( $n=4$ for each genotype and treatment). Paw thickness was significantly increased in both genotypes after CFA injection from $4 \mathrm{~h}$ to day 6, compared to saline injected mice. (B) Paw withdrawal latencies to heat after CFA or saline injection in WT and 5-HTT-/- mice $(n=4$ for each genotype and treatment). Paw withdrawal latencies to heat were significantly decreased in WT mice from $4 \mathrm{~h}$ to day 5 after CFA injection, compared to saline injected mice. In 5-HTT-/- mice, differences between CFA and saline were only significant at $4 \mathrm{~h}$ and 1 day after injection. (C) Mechanical withdrawal threshold after CFA or saline injection in WT and 5-HTT-/- mice ( $n=4$ for each genotype and treatment). Thresholds were significantly reduced in both genotypes after CFA injection compared with saline injection. (D) Bar graph showing withdrawal latencies to heat after CFA injection in 5-HTT-/- mice $(n=8)$. An injection of 5-HT $(5 \mu \mathrm{g}, n=4)$ but not of normal saline $(n=4)$ led to reduced withdrawal latencies in the 5HTT-/- mice, indicating thermal hyperalgesia $\left({ }^{*} p<0.05\right.$ vs. saline). Asterisks $\left({ }^{*}\right)$ indicate significant differences between CFA and saline injected mice in A-C, and between 5-HT and saline injected mice in D, $p<0.05$. \# indicates a significant difference between CFA-treated wild type and 5-HTT-/mice in $\mathrm{B}$.

hairs decreased after i.pl. CFA injection without differences between genotypes (Fig. 1C).

\subsection{5-5-HTT-/- mice have reduced levels of 5-HT}

After saline and CFA injection, 5-HT levels were lower in the L4/5 spinal cord, in the sciatic nerve and in adrenal glands of 5-HTT-/- mice compared with wild type mice at all time points examined ( $p<0.001$, Fig. 2$)$. Thus, the difference between genotypes was independent of the experimental treatment. 5-HT was further reduced in the $\mathrm{L} 4 / 5$ spinal cord in both genotypes on day 2 after CFA injection (Fig. 2A).

\subsection{Thermal hyperalgesia after CFA can be reestablished in 5-HTT-/- mice by i.pl. injection of 5-HT}

To test the assumption that the reduced thermal hyperalgesia in 5-HTT-/- mice was causally related to the reduced tissue 5-HT levels, we injected 5-HT or saline into the hindpaws of CFA-treated 5-HTT-/- mice $(n=4$ each) on day 4 , when withdrawal latencies were back to normal. When tested 12 and $60 \mathrm{~min}$ after 5-HT injection, 5-HTT-/- mice had reduced withdrawal thresholds to heat $(p<0.001)$, which had resolved by $24 \mathrm{~h}$ later (day 5). Saline injection on day 4 did not change withdrawal thresholds (Fig. 1D).

\subsection{Intraepidermal innervation is reduced after i.pl. $C F A$ in 5-HTT-/- mice}

Having seen reduced pain behaviour upon thermal stimuli in 5-HTT-/- mice after i.pl. CFA, we asked whether this was associated with differences in the local tissue reaction to CFA between genotypes. H\& E stains and immunostaining for macrophages revealed a moderate amount of inflammatory cells in skin of CFA-injected mice without differences between genotypes (Fig. 3A and B). PGP 9.5 immunoreactivity was used to visualize all intraepidermal nerve fibres (IENF). Numbers of IENF $/ \mathrm{mm}$ in saline injected animals were not different between genotypes. After i.pl. CFA injection, fibre numbers were only slightly reduced in wild type mice on day 7 ( $p<0.05$, Fig. $3 \mathrm{C}$ and E). In contrast, 

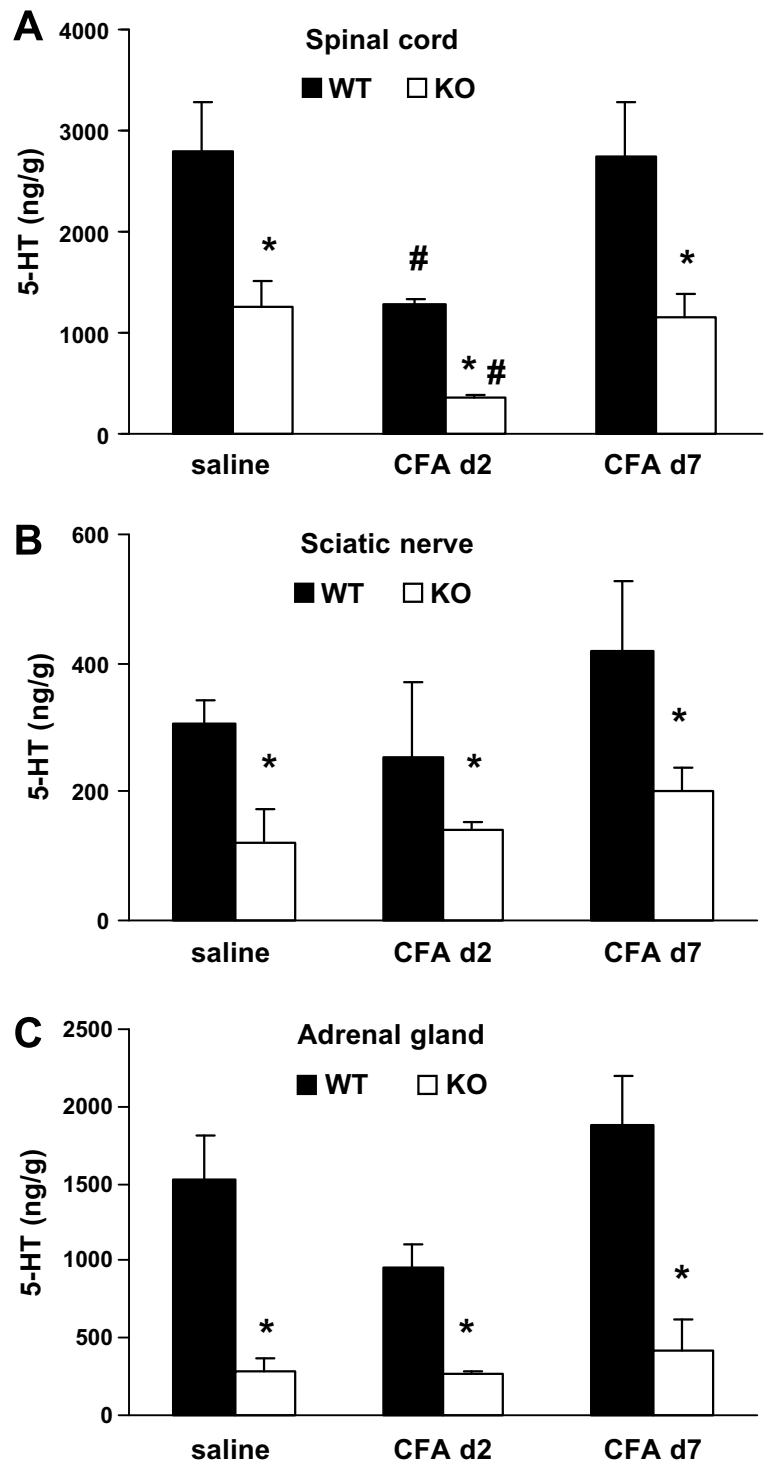

Fig. 2. 5-HT concentrations in spinal cord (A), sciatic nerve (B) and adrenal gland (C) tissue of 5-HTT-/- (KO) and wild type (WT) mice ( $n=4$ per genotype and treatment). ${ }^{*} p<0.05$ vs. WT., \# $p<0.05$ vs. saline. CFA d $2=2$ days after CFA injection; CFA d $7=7$ days after CFA injection.

5-HTT-/- mice showed a marked reduction in IENF density after i.pl. CFA on day 2 and 7 after CFA $(p<0.0005$, Fig. 3C and F). CGRP immunoreactivity was used to identify the subgroup of peptidergic sensory neurons. In saline injected mice, numbers of CGRP immunoreactive IENF $/ \mathrm{mm}$ were not different between genotypes. After i.pl. injection of CFA, wild type mice had unchanged CGRP immunoreactive fibre numbers but those in 5-HTT-/- mice were significantly decreased on day 2 and 7 after CFA ( $p<0.05$, Fig. 3D, G, H).

\subsection{ATF3 expression in DRG neurons}

Nuclear ATF3 staining was used to identify injured neurons (Tsujino et al., 2000). In saline injected mice, the rate of ATF3 immunoreactive DRG neurons was very low. After i.pl. CFA injection, the number of ATF3 positive nuclei increased significantly in both genotypes on day 7 , but considerably more in 5HTT-/- mice ( $p<0.005$, Fig. 4).

\section{Discussion}

Here we provide evidence that the 5-HTT-/- genotype in mice modulates inflammatory pain and we explore mechanisms underlying the behavioural characteristics of this genotype. Following i.pl. CFA injection, thermal hyperalgesia was reduced in 5-HTT-/- mice and could be reestablished by the i.pl. injection of 5-HT. Mechanical allodynia and paw swelling were unaltered in 5-HTT-/mice. 5-HT tissue levels after saline or CFA injections were lower in 5-HTT-/- mice than in wild type mice, and CFA injection reduced the spinal 5-HT content in both genotypes. Innervation of the plantar skin was reduced only in 5-HTT-/- mice after i.pl. CFA, which was in accordance with a higher number of injured DRG neurons as identified by ATF3 immunohistochemistry.

5-HTT-/- mice have been regarded as a model of life long administration of selective serotonin reuptake inhibitors (SSRI) (Bengel et al., 1998). Although 5-HT is widely accepted as an inflammatory mediator in the periphery (for review see (Sommer, 2006)), data on how SSRI influence 5-HT induced inflammation are controversial. The administration of a SSRI reduced inflammation in the carrageenan model (Bianchi et al., 1995; Abdel-Salam et al., 2004), but accelerated it in adjuvant arthritis (Harbuz et al., 1998). Here, we did not see an overt effect of the 5-HTT genotype on paw swelling. This may be due to the model (CFA vs. carrageenan) or to the fact that there are certainly many differences between 5-HTT-/- mice and mice with SSRI administration, including the long-term reduction in intracellular 5-HT in the knock-out mice (Bengel et al., 1998; Kim et al., 2001; Vogel et al., 2003).

As in previous studies, 5-HTT-/- mice had lower tissue contents of 5-HT than wild type mice (Bengel et al., 1998; Vogel et al., 2003). Low 5-HT in the peripheral nerve would be expected to lead to reduced thermal hyperalgesia. Indeed, we could rescue the hyperalgesic phenotype by exogenous application of 5-HT. The consequence of lowered spinal 5-HT is more difficult to interpret, since analgesic and pro-algesic spinal effects of 5-HT have been described (Suzuki et al., 2004). It was recently shown that depletion of endogenous spinal 5-HT attenuated hypersensitivity in a model of nerve injury pain (Rahman et al., 2006). Thus, low spinal 5HT as observed in 5-HTT-/- mice in the present study may also be related to the reduction in pain behaviour. However, without having established an adequate 

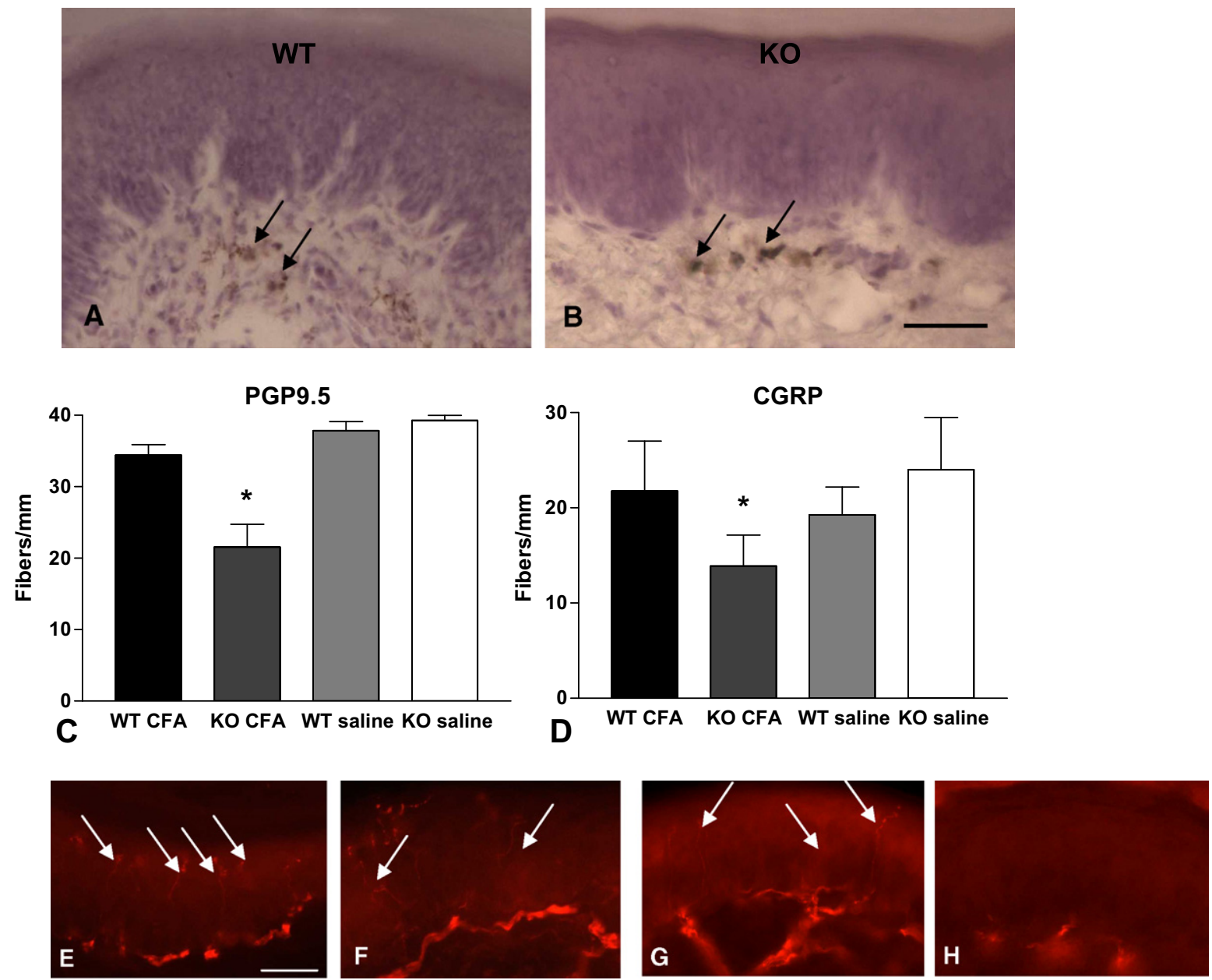

Fig. 3. Cryosections of plantar skin immunoreacted for macrophages from (A) wild type (WT) and (B) 5-HTT-/- (KO) mice. Numbers of PGP 9.5 (C) and CGRP (D) immunoreactive intraepidermal nerve fibres (IENF) per mm of plantar skin in KO and WT mice $(n=4$ per genotype and treatment) on day 2 and 7 after CFA injections compared with saline. ${ }^{*} p<0.05,{ }^{* *} p<0.0005$ vs. saline. E-H: Cryosections of plantar skin immunoreacted for PGP 9.5 (E, F) or CGRP $(\mathrm{G}, \mathrm{H})$ in WT $(\mathrm{E}, \mathrm{G})$ and $\mathrm{KO}(\mathrm{F}, \mathrm{H})$ mice $7 \mathrm{~d}$ after CFA injection. Note higher density of IENF (arrows) in skin from WT mice. Bars $=20 \mu \mathrm{m}$.

method to reconstitute spinal 5 -HT in an atraumatic way, this assumption cannot be confirmed. Additionally, one of the mediators of the indirect actions of 5-HT on pain, like prostaglandins (Tambeli et al., 2006) might be involved and might be present to a lesser degree in the inflammatory tissue of 5-HTT-/- mice. Although we found no differences in paw swelling, and thus no overt indication of a different degree of inflammation between genotypes, this possibility cannot be discarded.

As in our previous study using the CCI model of nerve injury (Vogel et al., 2003), we did not find an attenuation of mechanical withdrawal thresholds in the 5-HTT-/- mice. In contrast to thermal hyperalgesia, mechanical allodynia is thought to be mediated by Adelta-fibres and to depend on changes in spinal connectivity (Field et al., 1999). Thus, the 5-HTT-/- genotype appears to have more impact on peripherally generated pain than on central pain modulation, at least in this inflammatory model.
Nerve injury through CFA injection or through the subsequent inflammation has been anecdotally reported and nerve regeneration is impaired after previous CFA injection (Kato et al., 2003). We therefore investigated possible nerve injury by quantitation of IENFs and by ATF3 immunostaining of DRG neurons. Since IENF density was only slightly reduced and the number of ATF3 immunoreactive neurons was unaltered in wild type mice, we could not confirm a general nerve damaging action of CFA. However, numbers of total and of peptidergic IENFs were reduced after CFA in 5-HTT-/- mice, indicating a greater vulnerability of distal nerve endings in this genotype. This was corroborated by the finding of increased numbers of ATF3 immunoreactive nuclei in DRG neurons, showing that the peripheral insult indeed had an impact on the neuronal somata, although with a delayed time course. Thus, in 5HTT-/- mice sensory nerve fibres were more vulnerable to inflammation of their target tissues but the animals 

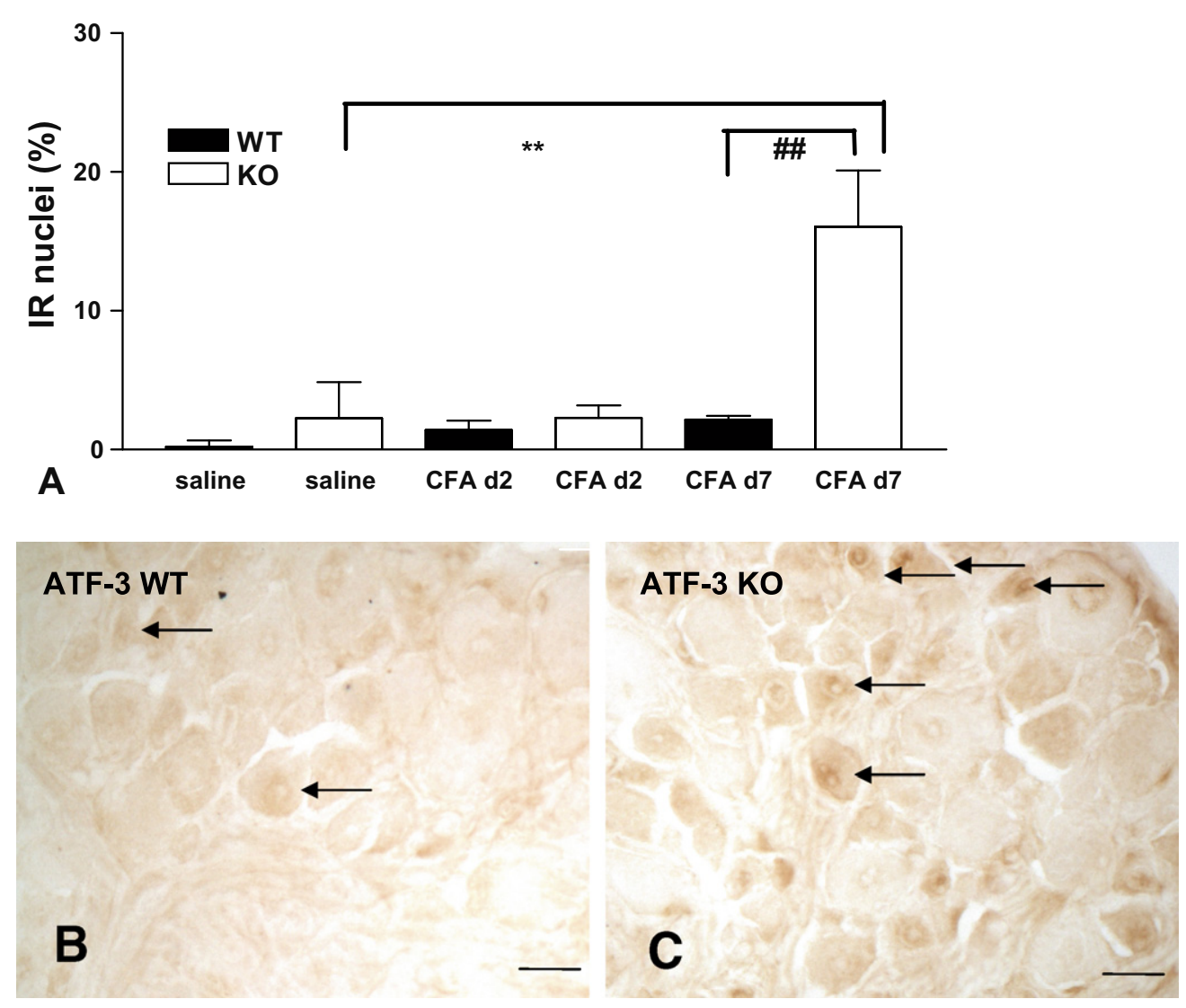

Fig. 4. (A) Percentage of DRG neuronal nuclei immunoreactive for ATF3. ${ }^{* *} p<0.005$ KO CFA d7 vs. KO saline and \#\# KO CFA d7 vs. WT CFA d7. CFA d $2=2$ days after CFA injection; CFA d7 $=7$ days after CFA injection. $N=4$ per genotype and treatment. B, C. Representative photomicrographs of frozen DRG sections immunoreacted for ATF3 in (B) wild type (WT) and (C) 5-HTT-/- (KO) mice $(n=4$ per genotype) after CFA injection. Arrows indicate neuronal profiles with ATF3-immunoreactive nuclei. Bar $=20 \mu \mathrm{m}$.

were less susceptible to thermal hyperalgesia. It is possible that the presence of fewer heat reactive neurons is one reason for reduced thermal hyperalgesia in the 5-HTT-/- mice. However, with the still large number of remaining IENFs, it is unlikely to be the sole cause of reduced heat pain, given that the correlation between IENF density and thermal withdrawal thresholds in mice is very low, at least in a nerve injury model (Lindenlaub et al., 2000). The reason for the increased axonal vulnerability is as yet unknown. It may be due to a lack of a trophic factor such as BDNF. BDNF expression is dependent on 5-HT function and is decreased by lower levels of 5-HT (Zetterstrom et al., 1999). Taken together, the 5-HTT-/- genotype protects from inflammatory thermal hyperalgesia, but entails greater peripheral nerve vulnerability upon inflammation. One might speculate that the human variants of 5-HTT genotypes (Murphy et al., 2004) might belong to the increasing number of variables recently detected to determine the extent of nerve lesions and the development of pain under the situation of a given nerve injury or inflammation (Tegeder et al., 2006; Üceyler et al., 2007).

\section{Acknowledgements}

This work was supported by the Deutsche Forschungsgemeinschaft (Sonderforschungsbereich 581), by the Interdisziplinäres Zentrum für Klinische Forschung (IZKF) Würzburg, and by the European Commission (NEWMOOD LSHM-CT-2003-503474). Barbara Dekant, Lydia Biko, Helga Brünner and Rainer Burger provided expert technical help.

\section{References}

Abdel-Salam OM, Baiuomy AR, Arbid MS. Studies on the antiinflammatory effect of fluoxetine in the rat. Pharmacol Res 2004;49:119-31.

Bengel D, Murphy DL, Andrews AM, Wichems CH, Feltner D, Heils A, et al. Altered brain serotonin homeostasis and locomotor insensitivity to 3, 4- methylenedioxymethamphetamine (Ecstasy) in serotonin transporter- deficient mice. Mol Pharmacol 1998;53:649-55.

Bennett GJ, Xie YK. A peripheral mononeuropathy in rat that produces disorders of pain sensation like those seen in man. Pain 1988;33:87-107.

Bianchi M, Rossoni G, Sacerdote P, Panerai AE, Berti F. Effects of chlomipramine and fluoxetine on subcutaneous carrageenininduced inflammation in the rat. Inflamm Res 1995;44:466-9. 
Chaplan SR, Bach FW, Pogrel JW, Chung JM, Yaksh TL. Quantitative assessment of tactile allodynia in the rat paw. J Neurosci Methods 1994;53:55-63.

Davis KD, Meyer RA, Campbell JN. Chemosensitivity and sensitization of nociceptive afferents that innervate the hairy skin of monkey. J Neurophysiol 1993;69:1071-81.

Dixon W. The up-and-down method for small samples. J Am Stat Assoc 1965;60:967-78.

Dray A. Inflammatory mediators of pain. Br J Anaesth 1995;75: $125-31$.

Eide PK, Hole K. The role of 5-hydroxytryptamine (5-HT) receptor subtypes and plasticity in the 5-HT systems in the regulation of nociceptive sensitivity. Cephalalgia 1993;13:75-85.

Fabre V, Beaufour C, Evrard A, Rioux A, Hanoun N, Lesch KP, et al. Altered expression and functions of serotonin 5-HT1A and 5HT1B receptors in knock-out mice lacking the 5-HT transporter. Eur J Neurosci 2000;12:2299-310.

Ferreira J, Campos MM, Pesquero JB, Araujo RC, Bader M, Calixto JB. Evidence for the participation of kinins in Freund's adjuvantinduced inflammatory and nociceptive responses in kinin B1 and B2 receptor knockout mice. Neuropharmacology 2001;41:1006-12.

Field MJ, Bramwell S, Hughes J, Singh L. Detection of static and dynamic components of mechanical allodynia in rat models of neuropathic pain: are they signalled by distinct primary sensory neurones? Pain 1999;83:303-11.

Harbuz MS, Marti O, Lightman SL, Jessop DS. Alteration of central serotonin modifies onset and severity of adjuvant-induced arthritis in the rat. Br J Rheumatol 1998;37:1077-83.

Hargreaves K, Dubner R, Brown F, Flores C, Joris J. A new and sensitive method for measuring thermal nociception in cutaneous hyperalgesia. Pain 1988;32:77-88.

Kato N, Nemoto K, Arino H, Fujikawa K. Influence of peripheral inflammation on growth-associated phosphoprotein (GAP-43) expression in dorsal root ganglia and on nerve recovery after crush injury. Neurosci Res 2003;45:297-303.

Kessler F, Falchetto R, Heim R, Meili R, Vorherr T, Strehler EE, et al. Study of calmodulin binding to the alternatively spliced C-terminal domain of the plasma membrane $\mathrm{Ca}^{2+}$ pump. Biochemistry 1992;31:11785-92.

Kim CH, Oh Y, Chung JM, Chung K. The changes in expression of three subtypes of TTX sensitive sodium channels in sensory neurons after spinal nerve ligation. Brain Res Mol Brain Res 2001;95:153-61.

Kim DK, Tolliver TJ, Huang SJ, Martin BJ, Andrews AM, Wichems $\mathrm{C}$, et al. Altered serotonin synthesis, turnover and dynamic regulation in multiple brain regions of mice lacking the serotonin transporter. Neuropharmacology 2005;49:798-810.

Lesch KP, Meyer J, Glatz K, Flugge G, Hinney A, Hebebrand J, et al. The 5-HT transporter gene-linked polymorphic region (5HTTLPR) in evolutionary perspective: alternative biallelic variation in rhesus monkeys. Rapid communication. J Neural Transm 1997;104:1259-66.

Lindenlaub T, Teuteberg P, Hartung T, Sommer C. Effects of neutralizing antibodies to TNF-alpha on pain-related behavior and nerve regeneration in mice with chronic constriction injury. Brain Res 2000;866:15-22.
Mathews TA, Fedele DE, Coppelli FM, Avila AM, Murphy DL, Andrews AM. Gene dose-dependent alterations in extraneuronal serotonin but not dopamine in mice with reduced serotonin transporter expression. J Neurosci Methods 2004;140:169-81.

McMahon SB, Cafferty WB, Marchand F. Immune and glial cell factors as pain mediators and modulators. Exp Neurol 2005; 192:444-62.

Moalem G, Grafe P, Tracey DJ. Chemical mediators enhance the excitability of unmyelinated sensory axons in normal and injured peripheral nerve of the rat. Neuroscience 2005;134:1399-411.

Montanez S, Owens WA, Gould GG, Murphy DL, Daws LC. Exaggerated effect of fluvoxamine in heterozygote serotonin transporter knockout mice. J Neurochem 2003;86:210-9.

Murphy DL, Lerner A, Rudnick G, Lesch KP. Serotonin transporter: gene, genetic disorders, and pharmacogenetics. Mol Interventions 2004;4:109-23.

Rahman W, Suzuki R, Webber M, Hunt SP, Dickenson AH. Depletion of endogenous spinal 5-HT attenuates the behavioural hypersensitivity to mechanical and cooling stimuli induced by spinal nerve ligation. Pain 2006;123:264-74.

Schäfers M, Geis C, Svensson CI, Luo ZD, Sommer C. Selective increase of tumour necrosis factor-alpha in injured and spared myelinated primary afferents after chronic constrictive injury of rat sciatic nerve. Eur J Neurosci 2003;17:791-804.

Sommer C. Is serotonin hyperalgesic or analgesic? Curr Pain Headache Rep 2006;10:101-6.

Sommer C, Schäfers M. Painful mononeuropathy in C57BL/Wld mice with delayed wallerian degeneration: differential effects of cytokine production and nerve regeneration on thermal and mechanical hypersensitivity. Brain Res 1998;784:154-62.

Suzuki R, Rygh LJ, Dickenson AH. Bad news from the brain: descending 5-HT pathways that control spinal pain processing. Trends Pharmacol Sci 2004;25:613-7.

Tambeli CH, Oliveira MC, Clemente JT, Pelegrini-da-Silva A, Parada CA. A novel mechanism involved in 5-hydroxytryptamine-induced nociception: The indirect activation of primary afferents. Neuroscience 2006;141:1517-24.

Tegeder I, Costigan M, Griffin RS, Abele A, Belfer I, Schmidt H, et al. GTP cyclohydrolase and tetrahydrobiopterin regulate pain sensitivity and persistence. Nat Med 2006;12:1269-77.

Tsujino H, Kondo E, Fukuoka T, Dai Y, Tokunaga A, Miki K, et al. Activating transcription factor 3 (ATF3) induction by axotomy in sensory and motoneurons: A novel neuronal marker of nerve injury. Mol Cell Neurosci 2000;15:170-82.

Üceyler N, Rogausch JP, Toyka KV, Sommer C. Differential expression of cytokines in painful and painless neuropathies. Neurology 2007;69:42-9.

Vogel C, Mössner R, Gerlach M, Heinemann T, Murphy DL, Riederer $\mathrm{P}$, et al. Absence of thermal hyperalgesia in serotonin transporterdeficient mice. J Neurosci 2003;23:708-15.

Zetterstrom TS, Pei Q, Madhav TR, Coppell AL, Lewis L, GrahameSmith DG. Manipulations of brain 5-HT levels affect gene expression for BDNF in rat brain. Neuropharmacology 1999;38:1063-73.

Zhang RX, Lao L, Wang X, Ren K, Berman BB. Electroacupuncture combined with indomethacin enhances antihyperalgesia in inflammatory rats. Pharmacol Biochem Behav 2004;78:793-7. 